\title{
Evaluation of wearable KPF goniometers in knee flexion-extension measurement for daily-life applications
}

\author{
Federico Lorussi \\ Research Center E.Piaggio \\ Largo L. Lazzarino 1 \\ 56126 Pisa, Italy \\ f.lorussi@ing.unipi.it
}

\author{
Nicola Carbonaro \\ Research Center E.Piaggio \\ Largo L. Lazzarino 1 \\ 56126 Pisa, Italy \\ n.carbonaro@ing.unipi.it \\ Alessandro Tognetti \\ Research Center E.Piaggio \\ Largo L. Lazzarino 1 \\ 56126 Pisa, Italy \\ a.tognetti@centropiaggio.unipi.it
}

\author{
Danilo De Rossi \\ Research Center E.Piaggio \\ Largo L. Lazzarino 1 \\ 56126 Pisa, Italy \\ d.derossi@centropiaggio.unipi.it
}

\begin{abstract}
Rehabilitation for stroke survivor is an important activity to recover daily-life functional performances. A daily life monitoring system composed of different subsystem was developed within the INTERACTION EU project. Sensing trousers using a couple of IMUs integrated on the thighs and shanks and a textile goniometers in the knee region was designed to detect kinematic asymmetries between affected and unaffected legs in gait/ambulation. In this study we compared the performances of our textile goniometer based on KPF materials with a couple of IMUs for the evaluation of knee flexion-extension. We used a statistical approach (T-student test) to verify that the signal gathered from the goniometer has the same information of the one extracted from the IMUs. These results demonstrate a reliable performance of our sensors, which have the advantage to be more flexible, lighter and less expensive than current wearable technologies.
\end{abstract}

\section{Keywords}

Wearable technologies, textile Goniometers, IMU

\section{INTRODUCTION}

Stroke survivors are trained to recover adequate control over their movements to optimise daily-life functional performances. Continuous daily-life monitoring of the functional activities of stroke patients in their physical interaction with the environment is essential for optimal guidance of rehabilitation therapy by medical professionals and coaching of the patient. Such performance information cannot be easily obtained in daily life conditions with current monitoring systems.
The objective of the INTERACTION EU project was to develop and validate an unobtrusive and modular sensing system for monitoring daily life activities of stroke subjects, evaluating their physical interactions with the environment and training motor function of upper and lower extremities. The system is unobtrusively integrated in clothing (e-textile) and includes fabric-based and distributed inertial sensing, providing tele-monitoring and adaptive on-body feedback capabilities [1]. Through this prototype it is possible to provide full body motion monitoring (upper and lower arms including hands and feet, trunk, center of mass) and to detect the patient kinetic interaction with the environment at the body extremities (sensing gloves and shoes). In particular, a set of wireless on-body devices fully integrated in a modular textile platform (shirt, trousers, gloves, shoes) were developed. From a technical point of view, the main objective of the project is to exploit smart sensory fusion of textile, flexible and inertial sensors in order to develop an effective wearable sensing system as much as possible unobtrusive to the user .

In this paper we report a study that compares the performances of a textile goniometer with a couple of Inertial measurement units (IMU) system in order to evaluate their performance in terms of evaluation of knee flexo-extension activities. Over the last decade, MEMS-based IMUS have been developed for use in human motion tracking. IMUs are widely considered as the gold standard in wearable motion sensing $[2,3,4]$. However for daily life applications, less obtrusive, less expensive and more easy to wear sensing technologies may be attractive. On these regards, previous studies described the use of textile integrated sensors for the development of wearable motion monitoring systems $[5,6$, 7]. This class of sensors are low cost, flexible, light and they can be perfectly adapted to subject's different body structures. However, their performance is limited to the reconstruction of wide and slow movements. Recently we have developed a new generation of textile goniometers having very promising performance in terms of angular measurement between consecutive body segments $[8,9,10]$. In this study we will compare these novel sensors with IMUs in knee flexion-extension activities. 


\section{MATERIALS AND METHOD}

One of the INTERACTION main requirement was to detect kinematic asymmetries between affected and unaffected legs in gait/ambulation. The sensing trousers were conceived to deal with this objective using a couple of IMUs integrated on the thighs and shanks [1], and a KPF goniometer in the knee region. In particular, the main idea was to use a data fusion of the information deriving from IMUs and KPF goniometers for robust knee flexion-extension estimation. Sensor fusion is defined as the combination of sensory data or data derived from disparate sources such that the resulting information is in some sense better than would be possible when these sources were used individually. The term better in this case can mean more accurate, more complete, or more dependable, or refer to the result of an emerging view. According to the given definition, sensor fusion strategies make sense only if they are applied to a redundant set of sensors. Choosing a particular problem of parameter estimation the application of sensor fusion may have two type of possible results: 1) Data output is more reliable with respect to the quality of measurement derived by the single class of sensors 2) Data output has the same reliability (within the aim of the measurement) of a subset of the actual sensor system, and the outputs can be reduced, in order to obtain a minimal, cheaper and unobtrusive measurement system.

In this study, data fusion has been conceived to combine information of a couple of IMUs, placed on the thigh bone and on the tibia, and a goniometer which directly measures the angle between the two bones. Remembering that the prototype will be used by post-stroke survivors, it would be very advantageous to reduce the overall system complexity and increasing the usability for the user, if we can demonstrate that IMUs information is not adding anything more than what we can retrieve from KPF goniometers. This will allow designers to remove the more expensive sensors obtaining a cheaper and more comfortable device. According to this aims, a statistical procedure, described in details in next section, was adopted proving that the addition of IMUs sensors does not improve the reliability of the textile goniometer for the evaluation of knee orientation.

\subsection{KPF Goniometers}

Textile goniometers are produced using knitted piezoresistive fabrics (KPF) [11]. The KPF goniometer structure is built up by attaching two identical KPF layer using an insulating adhesive membrane. Each piezoresistive layer can be represented as a series of three resistances. It has four semicircular pads for sensor wiring and for a four point measurement method. This electronic circuit topology (see figure 1) allows to minimize the effect of connection resistances. In particular, suppling a constant current I through the external pads the voltages $V_{L 1}\left(V_{B 1}-V_{B 2}\right)$ and $V_{L 2}\left(V_{B 3}-V_{B 4}\right)$ between the internal pads are measured. The acquisition system is designed with an high input impedance stage realized by two instrumentation amplifiers ( $A 1$ and $A 2)$ permits to read only the voltages across the relative piezoresistive sensors. So $V_{L 1}$ and $V_{L 2}$ are directly related to resistances $R_{L 1}$ and $R_{L 2}$ by means of the knowledge of $I$. A third differential amplifier $A 3$ has been included to amplify the difference $\left(V_{L 1}-V_{L 2}\right)$ which is proportional to the $\Delta R_{D L}$ that is related to the bending angle. This last stage is used to adapt the signal to the input range of the ADC converter and to compensate the goniometer offset value. Considering a)

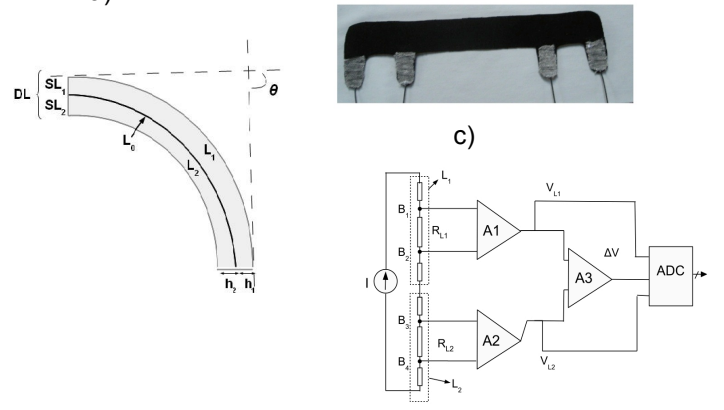

Figure 1: KFP Gonomieters working principle. a) It is represented the double layer configuration and the angle measured during bending. b) A picture of the a KPF goniometer is reported showing the four pads for wiring connection. c) Schematic block of the electronic front-end designed for KPF goniometers measurement.

the described front-end and imposing the same amplification for $A 1$ and $A 2$, the $\Delta V$ gathered is proportional to the bending angle $(\theta)$. The sensor can be calibrated associating the output voltage to two different angular positions: typically at 0 degrees (i.e. to obtain offset) and at a second angular position $\theta_{C}$ (i.e. to obtain the sensitivity $a=\frac{V_{\theta_{c}}-b}{\theta_{C}}$ ), as described in $[8,10]$.

\subsection{Experimental Set-up}

The experimental set-up is reported in figure 2. A double layer KPF goniometer was applied to a knee band and compared with the outputs of two IMUs (MTw provided by XSENS [12] ) placed on the thigh and on the calf, and used as a gold standard measurement instrumentation. The goniometer $(40 \mathrm{~cm}$ length and $2.5 \mathrm{~cm}$ width, distance between the internal pads $30 \mathrm{~cm}$ ), was used to entirely cover the knee joint. IMU-based joint kinematic estimations, widely described in [2], have a reconstruction accuracy that is lower than $3^{\circ}$ for flexion-extension joint movements [13], making the good agreement of our comparison test very promising. Data have been acquired on different motor tasks, such as flexion-extension in monopodalic contralateral standing position and during activities like slow, medium and fast speed walking (running). Described experiments have been acquired for five times per trial on different tests. The $\mathrm{KPF}$ goniometer was calibrated in order to measure zero degrees when the knee was completely extended. Regarding IMUs system, the flexion-extension knee angle was elaborated taking into account the components of the rotation matrix which describe the orientation of the IMU frame on the calf with respect to the frame of the IMU placed on the thigh.

\section{TEST AND RESULTS}

The knee flexion-extension measured with the KPF goniometer $\theta_{g}(t)$ was compared with the angle obtained by the two IMUs $\theta_{I M U}(t)$. A first graphical evaluation of the system performances in comparison is given by the following figures. The following step in data analysis consisted in accomplish- 


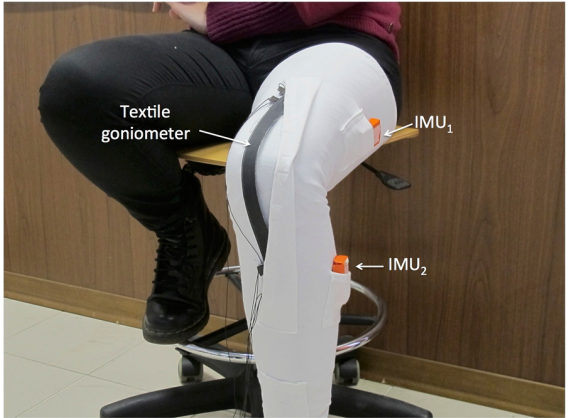

Figure 2: Prototype used for experimental set-up: KPF goniometer applied to a band for the detection of knee flexion/extension; two IMUs fixed in the thigh and on the calf.

a)

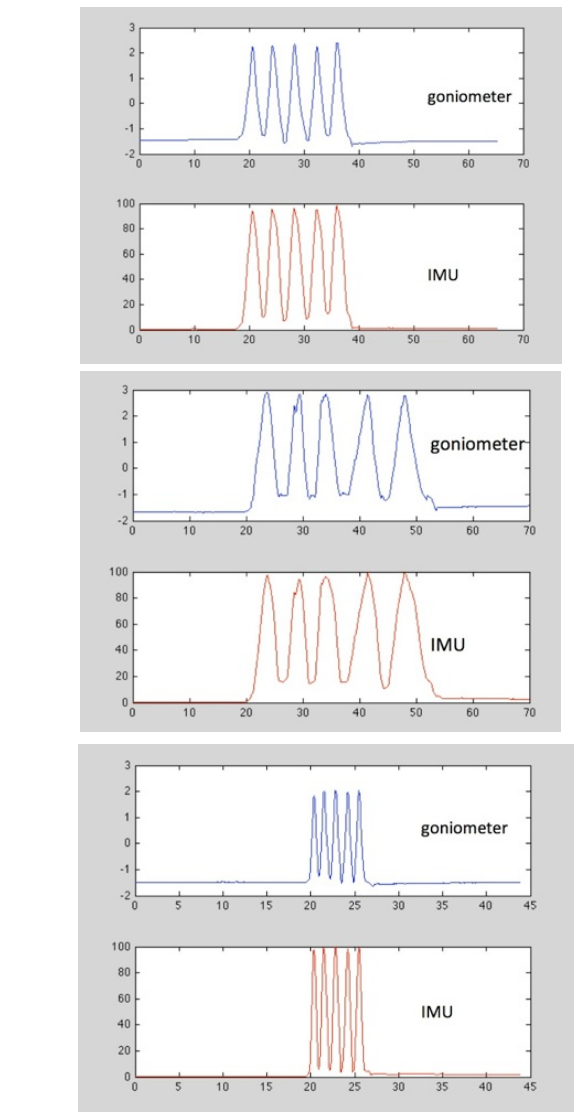

b)

Figure 3: Comparison between goniometers and IMUs in knee flexion extension detection during monopodalic standing. In a) slow knee flexionextensions are performed, in b) with medium speed and in c) user performed the activities with fast speed knee flexion-extension.

ing a statistical inferential T-test in order to determine if the samples obtained by the two different measurement system $\theta_{g}(t)$ and $\theta_{I M U}(t)$ belong to the same population. The two statistics of (1) and (2) were used to perform an inferential

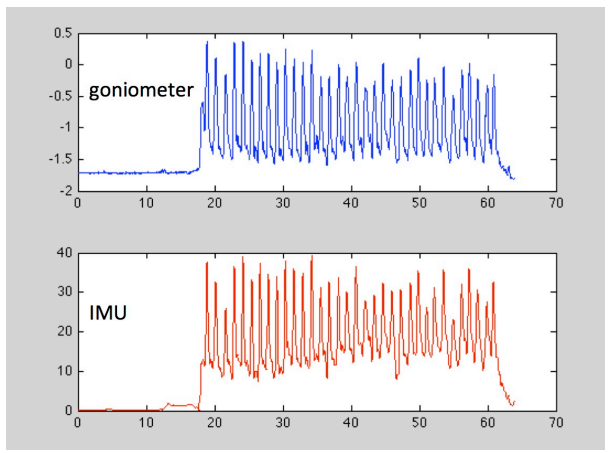

Figure 4: Comparison between goniometers and IMUs in knee flexion extension detection during walking activities performed with different speed. In figure results of only medium speed walking activities are reported.

Table 1: Statistics on differences between IMU and goniometer behaviour. $t$ represent the student statistic, $\mathrm{p}$ is the related $\mathrm{p}$-value.

\begin{tabular}{|l|c|c|c|c|c|}
\hline \multicolumn{1}{|c|}{ Activity } & $\mathbf{X}$ & $\sigma$ & $\mathbf{t}$ & $\mathbf{p}$ & Verified \\
\hline $\begin{array}{l}\text { Slow } \\
\text { flexion }\end{array}$ & 0.06 & 0.28 & -1.56 & 0.33 & $\mathrm{Y}$ \\
\hline Normal flexion & -0.04 & 0.33 & 0.93 & 0.36 & $\mathrm{Y}$ \\
\hline Fast flexion & 0.08 & 0.40 & -1.63 & 0.20 & $\mathrm{Y}$ \\
\hline Slow walking & 0.07 & 0.30 & -1.61 & 0.26 & $\mathrm{Y}$ \\
\hline Normal walking & -0.06 & 0.39 & 0.89 & 0.28 & $\mathrm{Y}$ \\
\hline Running & 0.12 & 0.45 & 1.61 & 0.25 & $\mathrm{Y}$ \\
\hline
\end{tabular}

comparison between the measurement systems.

$$
\begin{gathered}
X=\overline{\theta_{I M U}-\theta_{g}}=\frac{1}{t_{1}-t_{0}} \int_{t_{0}}^{t_{1}} \theta_{I M U}(t)-\theta_{g}(t) d t \\
\sigma=\left\|\theta_{I M U}-\theta_{g}\right\|_{2}=\frac{1}{t_{1}-t_{0}} \sqrt{\int_{t_{0}}^{t_{1}}\left(\theta_{I M U}(t)-\theta_{g}(t)\right)^{2} d t .}
\end{gathered}
$$

Supposing that $\theta_{I M U}(t)$ and $\theta_{g}(t)$ are two random variables, the difference $\theta_{g}(t)-\theta_{I M U}(t)$ has to be a random variable also. If we prove that $\mathrm{X}$ is a zero-mean random variable which respect to the chosen confidence $\sigma$, we have proven that the system is redundant and a sensor can be removed. In practice, the described test (performed as a parametric T-test) discriminates if the data relieved by using the goniometer belongs to the population of the trials performed by using the couple of IMUs or not. In case the trial fails, a fusion algorithm (e.g. Kalman based estimator, where a type of sensor is used in the prediction phase) has to be implemented since the complete system provides a more accurate information than the single part. Fixed a significance level $\sigma=0.05$ the t-tests performed on the zero mean variable $\mathrm{X}$ associated to the standard deviation $\sigma$ produced the results included in Table 1 . In each of the examined cases the Zero-Hypothesis is verified, i.e. the measurement instrumentation provide the same information with the chosen confidence. Considering the prototype we have developed within the Interaction project [1], this implies that, from a minimalsensor-system point of view, the IMU on the calf does not provide any additional information on the knee flexion, and, 
could be eventually removed, if the IMU placed on the shoe provides information on the knee torsion (no movements on the horizontal plane are allowed by the ankle and the 3D info on the feet permits to find out the intra-extra rotation of the femoral-tibial joint). Then a comparison between the goniometer information and a sensor fusion of data coming from IMUs and textile goniometer was performed. A sensor fusion algorithm based on a Kalman filter was adopted using the output of the goniometer as the current status of the system and the value of the angle derived by the two IMUs as a predictor. This further treatment allows to determine if combining the data of IMUs and goniometers would improve the estimation of knee orientation. For graphical simplicity we include only the result, as an example, of the data fusion realized on the slow knee flexion extension (Figure 5) where the green graph represents the filtered data. As it is

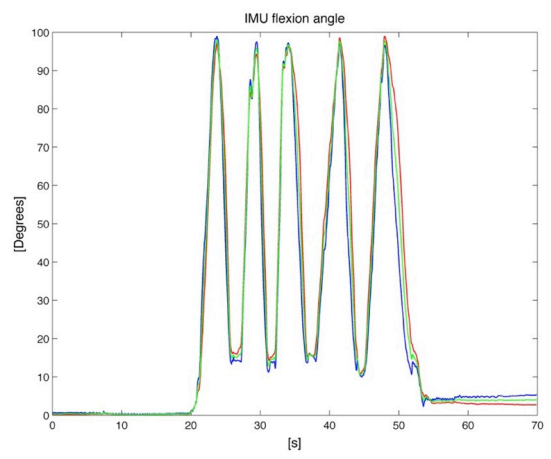

Figure 5: Mixed data in knee flexion-extension. Signal represented in blue marks the goniometer angle trend, while the red one trace the output of the IMUs. Mixed data are represented by the green graph.

possibile to note also from Figure 5 the evaluation of knee flex-extension extracted from the combination of data coming from the two measurament system is almost equal to the one detected using only the KPF goniometer.

\section{CONCLUSIONS}

In this study we reported the capability of textile goniometer based on KPF materials to be robust and reliable for the evaluation of knee flexion-extension. We performed a comparison with widely accepted wearable measurement systems (IMUs) and we were able to demonstrate a reliable performance of our sensors, which have the advantage to be more flexible, lighter and less expensive than current wearable technologies. This preliminary test has shown the potentiality to use textile goniometers in daily life monitoring of gait and balance of stroke survivors and of other relevant populations. Further studies are on going with particular focus on extensive testing on patients and integration with other sensors such as sensing shoes and insoles.

\section{ACKNOWLEDGMENTS}

This research has been supported by the EU 7th framework project INTERACTION (FP7-ICT-2011-7-287351).

\section{REFERENCES}

[1] A. Tognetti, F. Lorussi, N. Carbonaro, D. De Rossi, G. De Toma, C. Mancuso, R. Paradiso, H. Luinge, J. Reenalda, E. Droog, et al., "Daily-life monitoring of stroke survivors motor performance: the interaction sensing system," in Engineering in Medicine and Biology Society (EMBC), 2014 36th Annual International Conference of the IEEE, pp. 4099-4102, IEEE, 2014.

[2] H. J. Luinge and P. H. Veltink, "Measuring orientation of human body segments using miniature gyroscopes and accelerometers," Medical and Biological Engineering and computing, vol. 43, no. 2, pp. 273-282, 2005.

[3] K. Liu, T. Liu, K. Shibata, Y. Inoue, and R. Zheng, "Novel approach to ambulatory assessment of human segmental orientation on a wearable sensor system," Journal of biomechanics, vol. 42, no. 16, pp. 2747-2752, 2009.

[4] H. Zhou and H. Hu, "Human motion tracking for rehabilitationâĂTa survey," Biomedical Signal Processing and Control, vol. 3, no. 1, pp. 1-18, 2008.

[5] D. De Rossi, F. Carpi, F. Lorussi, R. Paradiso, E. Scilingo, and A. Tognetti, "Electroactive fabrics and wearable man-machine interfaces," Wearable Electronics and Photonics, pp. 59-80, 2005.

[6] N. Vanello, V. Hartwig, M. Tesconi, E. Ricciardi, A. Tognetti, G. Zupone, R. Gassert, D. Chapuis, N. Sgambelluri, E. P. Scilingo, et al., "Sensing glove for brain studies: design and assessment of its compatibility for fmri with a robust test," Mechatronics, IEEE/ASME Transactions on, vol. 13, no. 3, pp. 345-354, 2008.

[7] P. T. Gibbs and H. H. Asada, "Wearable conductive fiber sensors for multi-axis human joint angle measurements," Journal of NeuroEngineering and Rehabilitation, vol. 2, no. 1, p. 7, 2005.

[8] A. Tognetti, F. Lorussi, G. D. Mura, N. Carbonaro, M. Pacelli, R. Paradiso, and D. D. Rossi, "New generation of wearable goniometers for motion capture systems," Journal of neuroengineering and rehabilitation, vol. 11, no. 1, p. 56, 2014.

[9] N. Carbonaro, G. D. Mura, F. Lorussi, R. Paradiso, D. De Rossi, and A. Tognetti, "Exploiting wearable goniometer technology for motion sensing gloves," Biomedical and Health Informatics, IEEE Journal of, vol. 18, no. 6, pp. 1788-1795, 2014.

[10] G. Dalle Mura, F. Lorussi, A. Tognetti, G. Anania, N. Carbonaro, M. Pacelli, R. Paradiso, and D. De Rossi, "Piezoresistive goniometer network for sensing gloves," in XIII Mediterranean Conference on Medical and Biological Engineering and Computing 2013, pp. 1547-1550, Springer, 2014.

[11] M. Pacelli, L. Caldani, and R. Paradiso, "Textile piezoresistive sensors for biomechanical variables monitoring," in Engineering in Medicine and Biology Society, 2006. EMBS'06. 28th Annual International Conference of the IEEE, pp. 5358-5361, IEEE, 2006.

[12] "Xsens." www.xsens.com/en/general/mtw.

[13] P. Picerno, A. Cereatti, and A. Cappozzo, "Joint kinematics estimate using wearable inertial and magnetic sensing modules," Gait \& posture, vol. 28, no. 4, pp. 588-595, 2008. 